\title{
Prompt use of mechanical cardiopulmonary resuscitation in out-of-hospital cardiac arrest: the MECCA study report
}

Venkataraman Anantharaman ${ }^{1}$, MBBS, FRCPEd, Boon Lui Benjamin $\mathrm{Ng}^{2}$, MBBS, MSc, Shiang Hu Ang ${ }^{3}$, MBBS, MRCSEd, Chun Yue Francis Lee ${ }^{4}$, MBBS, FRCSEd, Siew Hon Benjamin Leong ${ }^{5}$, MBBS, MRCSEd, Marcus Eng Hock Ong ${ }^{1,6}$, MBBS, MPH,

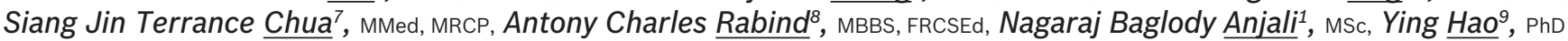

INTRODUCTION Early use of mechanical cardiopulmonary resuscitation (CPR) for out-of-hospital cardiac arrest (OHCA) may improve survival outcomes. Current evidence for such devices uses outcomes from an intention-to-treat (ITT) perspective. We aimed to determine whether early use of mechanical CPR using a LUCAS 2 device results in better outcomes.

METHODS A prospective, randomised, multicentre study was conducted over one year with LUCAS 2 devices in 14 ambulances and manual CPR in 32 ambulances to manage OHCA. The primary outcome was return of spontaneous circulation (ROSC). Secondary outcomes were survival at 24 hours, discharge from hospital and 30 days.

RESULTS Of the 1,274 patients recruited, 1,191 were eligible for analysis. 889 had manual CPR and 302 had LUCAS CPR. From an ITT perspective, outcomes for manual and LUCAS CPR were: ROSC $29.2 \%$ and $31.1 \%$ (odds ratio [OR] 1.09, 95\% confidence interval [Cl] 0.82-1.45; $\mathrm{p}=0.537)$; 24-hour survival $11.2 \%$ and $13.2 \%(\mathrm{OR} 1.20,95 \% \mathrm{Cl} 0.81-1.78$; $\mathrm{p}=0.352$ ); survival to discharge $3.6 \%$ and $4.3 \%$ (OR $1.20,95 \% \mathrm{Cl} 0.62-2.33 ; \mathrm{p}=0.579$ ); and 30 -day survival $3.0 \%$ and $4.0 \%$ (OR 1.32, 95\% Cl 0.66-2.64; $\mathrm{p}=0.430$ ), respectively. By as-treated analysis, outcomes for manual, early LUCAS and late LUCAS CPR were: ROSC 28.0\%, 36.9\% and 24.5\%; 24-hour survival $10.6 \%, 15.5 \%$ and $8.2 \%$; survival to discharge $2.9 \%, 5.8 \%$ and $2.0 \%$; and 30 -day survival $2.4 \%, 5.8 \%$ and $0.0 \%$, respectively. Adjusted OR for survival with early LUCAS vs. manual CPR was 1.47 after adjustment for other variables $(p=0.026)$.

CONCLUSION This study showed a survival benefit with LUCAS CPR as compared to manual CPR only, when the device was applied early on-site.

Keywords: cardiac arrest, LUCAS, mechanical CPR, return of spontaneous circulation, survival to discharge

\section{INTRODUCTION}

The current consensus on the science of resuscitation ${ }^{(1)}$ accepts that return of spontaneous circulation (ROSC) in a cardiac arrest patient would most likely be achieved with a combination of early, consistent administration of high-quality chest compressions of about $5.0 \mathrm{~cm}$ in adults and at a rate of 100-120 per minute, with full chest recoil after each compression. Providing all these early in the cardiac arrest management cycle is a major challenge in most communities. In highly urbanised communities, such as Singapore, where more than $80 \%$ of the residents live and work in high-rise buildings, it is even more challenging to provide such high-quality resuscitation in addition to the handicap of longer access time for emergency medical services and longer evacuation times. Furthermore, many of such communities have relatively low bystander cardiopulmonary resuscitation (CPR) rates $^{(2)}$ of around $20 \%-25 \%$, and would thus require more urgently the early use of effective CPR to enhance out-of-hospital cardiac arrest $(\mathrm{OHCA})$ survival.

There is increasing realisation that the quality of manual CPR may not be adequate after the first few minutes of initiation, especially with the realities of inconsistent rate, early onset of rescuer fatigue, ${ }^{(3)}$ and the near-impossibility of providing uninterrupted compressions while a casualty is being moved from the location of arrest to the hospital - carried down staircases and elevators, loaded onto waiting ambulances and then driven rapidly through roads and junctions. Over the last few decades, the development of the mechanical chest compression device has been seen as a potential solution for better outcomes. These devices have demonstrated improved cardiovascular haemodynamics, ${ }^{(4)}$ in terms of usage and survival, especially in laboratory environments. In laboratory-based trials, mechanical and manual CPR would usually have been initiated after a cardiac arrest time of 1-10 minutes, and the outcomes assessed shortly thereafter.

Early pilot studies ${ }^{(5,6)}$ comparing high-quality standard manual CPR with a load-distributing band (LDB) mechanical CPR device (also called the AutoPulse) demonstrated that the latter significantly improved haemodynamics as well as coronary and cerebral blood flow, with the potential for improved neurologically intact survival. In patients with OHCA, the LDB-CPR device has

\footnotetext{
${ }^{1}$ Department of Emergency Medicine, Singapore General Hospital, ${ }^{2}$ Office of Clinical Governance, Tan Tock Seng Hospital, ${ }^{3} \mathrm{Accident}$ and Emergency Department, Changi General Hospital, ${ }^{4}$ Acute and Emergency Care, Khoo Teck Puat Hospital, ${ }^{5}$ Emergency Medicine Department, National University Health System, ${ }^{6} \mathrm{Health}$ Services and Systems Research, Duke-NUS Medical School, ${ }^{7}$ Department of Cardiology, National Heart Centre Singapore, ${ }^{8}$ Accident and Emergency Department, Ng Teng Fong General Hospital, ${ }^{9} \mathrm{Health}$ Services Research Unit, Singapore General Hospital, Singapore

Correspondence: Prof Venkataraman Anantharaman, Senior Consultant, Department of Emergency Medicine, Singapore General Hospital, Outram Road, Singapore 169608.
} anantharaman@singhealth.com.sg 
also been demonstrated to increase systolic and diastolic blood pressures compared to manual chest compressions. ${ }^{(7)}$

However, clinical trials in this area have shown mixed results. A four-year, before-after study conducted from 2001 to 2005 showed that cardiac arrest patients managed with LDB-CPR ( $n=284)$ compared to those managed with standard manual CPR $(\mathrm{n}=499)$ had a higher survival to hospital discharge rate (9.7\% vs. $2.9 \%$; adjusted odds ratio [OR] 2.27 ; $95 \%$ confidence interval $[\mathrm{CI}] 1.11-4.77)$. The study also noted better mechanical CPR performance if ambulance response time was less than eight minutes. ${ }^{(8)}$ Another study of 1,011 patients with OHCA, of whom 552 received LDB-CPR after evacuation to hospital and 459 continued to receive manual $C P R$, showed a higher rate of survival to hospital discharge in the LDB-CPR group (3.3\% vs. $1.3 \%$; adjusted OR 1.42; 95\% Cl 0.47-4.29). The LDB group also had more survivors with better cerebral performance category scores. ${ }^{(9)}$

The other commonly used mechanical CPR device is the LUCAS (Lund University Cardiopulmonary Assist System; Jolife $A B$, Lund, Sweden), which has been tested in laboratory-based animal and human experiments. ${ }^{(10-12)}$ These studies demonstrated higher cardiac output, carotid artery blood flow, end-tidal carbon dioxide, aortic and coronary perfusion pressures, and ROSC rates with mechanical rather than manual CPR, although both were initiated at about the same time. The LUCAS device has also been used effectively during performance of advanced resuscitation techniques. ${ }^{(13-17}$

A 2006 study that compared the use of the LUCAS device and standard manual CPR in 328 OHCA patients showed that an equal proportion of patients achieved ROSC (51\%) and were discharged alive from hospital ( $8 \%$ vs. $10 \%$ ); however, there was a mean delay of eight minutes in the application of the mechanical device as opposed to the start of manual CPR. ${ }^{(18)}$ In a 2009 trial involving $126 \mathrm{OHCA}$ patients, Axelsson et al showed that the LUCAS device generated higher initial, minimum and average values of partial pressure of end-tidal carbon dioxide compared with manual CPR. ${ }^{(12)}$

Two large trials ${ }^{(19-20)}$ on mechanical CPR were carried out in the last decade. The first is the CIRC trial, ${ }^{(19)}$ which showed that the use of the AutoPulse was at least equivalent to very good quality manual CPR, although it did not report time delays in application of the AutoPulse on the patients. The other large study, with 2,589 patients, was the LINC trial, ${ }^{(20)}$ which found no significant difference in four-hour survival between LUCAS and manual CPR. In the latter study, there was an average four-minute delay in application of the mechanical device. The delayed initiation of the LUCAS device raises the issues of questionable quality of CPR from first contact to start of mechanical CPR and the potential of using this intervention on a higher-risk group with a lower chance of survival.

While it is generally believed that survival in cardiac arrest is highly dependent on early application of good-quality CPR, it would be reasonable to expect that, even with mechanical CPR, early application and use of the device would be more likely to show benefit than late application. Mechanical chest compression devices were developed to assist rescuers in giving consistent high-quality compressions. It is therefore important to determine whether there would be any differences in outcome if mechanical devices were applied at approximately the same time as manual CPR for patients sustaining OHCA.

The current randomised MECCA (Mechanical Cardiopulmonary Resuscitation Versus Standard Manual CPR in Out-of-Hospital Cardiac Arrest by Emergency Ambulance Crew) study compares the outcomes of mechanical CPR (i.e. LUCAS 2) and standard manual CPR when used by emergency ambulance crew in the outof-hospital environment, especially when the timing of initiation of either CPR method was similar.

\section{METHODS}

\section{Study description}

MECCA was a randomised controlled trial on out-of-hospital use of mechanical versus manual CPR for the management of adult OHCA. Waiver of informed consent for the study was approved by the institutional review boards (IRBs) of the two sponsoring institutions, National Healthcare Group (NHG) research cluster and Singapore Health Services (SingHealth) research cluster. The following were the six hospitals where the cardiac arrest casualties were evacuated to: NHG - Tan Tock Seng Hospital, Khoo Teck Puat Hospital, National University Hospital and Jurong General Hospital; and SingHealth - Singapore General Hospital and Changi General Hospital. The patients were initially attended to by the ambulance crew of Singapore's public Emergency Ambulance Service.

\section{Crew training and proficiency}

The ambulance crew underwent four hours of intensive training on the use of the LUCAS 2 device (Physio-Control, Redmond, Washington, USA). Prior to that, they also received revision on the performance of standard manual 30:2 CPR, as prescribed by the 2010 guidelines of the International Liaison Committee on Resuscitation (ILCOR) and the 2011 guidelines of the National Resuscitation Council, Singapore, a member of the Resuscitation Council of Asia, which is part of ILCOR. The ambulance crew were required to demonstrate proficiency in the skills of manual and LUCAS 2 CPR in a classroom environment, as well as continued proficiency at three-monthly intervals through supervised performance in classroom environments and production of rhythm strips.

\section{Randomisation of ambulances and allocation of calls}

Randomisation of the 46 ambulances in the Emergency Ambulance Service was done prior to the start of the trial: 14 ambulances were allocated to the LUCAS 2 arm and the remaining 32 to the manual CPR arm. Randomisation was carried out in this manner, as only 14 LUCAS 2 devices were available for the trial. At the start of the trial, only 12 of the 14 ambulances were fitted with the LUCAS 2 device; the remaining two devices arrived two months later and were then placed in the other two pre-allocated ambulances. Assignment of calls to the ambulances was made by call centre staff, who were blinded to the LUCAS 2 allocations. However, the care providers and hospital staff who 
received the casualties could not be blinded to the treatment arms, for obvious reasons.

\section{Data and safety monitoring}

An independent data and safety monitoring board monitored the trial at three and six months after the study onset to determine whether the pre-defined trial termination criteria were met.

\section{Inclusion and exclusion criteria}

From 23 May 2011 to 22 May 2012, all patients aged $\geq 21$ years with an $\mathrm{OHCA}$ of presumed cardiac origin and who were attended to by the ambulance crew of the Emergency Ambulance Service were included in the study. Patients were excluded if they were $<21$ years old, prisoners, presumed to be pregnant or cases of trauma arrest.

\section{Procedures undertaken during the trial}

The emergency ambulance crew used manual 30:2 CPR soon after arriving at the patient's side. For patients managed by LUCAS-allocated ambulances, the LUCAS 2 device was applied soon after manual CPR had begun. The device was set to provide 100 compressions per minute continuously. At every tenth compression, the ambulance crew would deliver one ventilation with a bag-valve-mask device. Chest compressions by the LUCAS 2 device were not interrupted during ventilation or delivery of defibrillator shocks. For patients managed by manual CPR-randomised ambulances, 30:2 CPR would continue till the casualties were handed over to the staff of the receiving hospital emergency department (ED). The timings of initiation of manual and mechanical CPR were documented. Defibrillation, airway management, ventilation through a laryngeal mask airway and intravenous epinephrine were also provided as required. The casualty was initially evacuated to the ambulance and subsequently sent to the nearest public general hospital. The staff of the ED managed the patient according to the respective departmental protocols.

\section{Data collection}

Data was collected from ambulance and hospital patient care records, and entered first into an anonymised case record form and then an electronic database. The data was maintained by a research coordinator and a trial statistician.

\section{Outcome measures}

The primary outcome measure was ROSC. Secondary outcome measures were survival at 24 hours, hospital discharge and 30 days. The IRBs of the participating hospitals approved the trial and provided complete waiver of informed consent. Community consultation was conducted through public fora, briefings to the print and broadcast media, and a period for public feedback before final IRB approval was granted.

\section{Statistical analysis}

Based on the need to detect a $20 \%$ improvement in ROSC between mechanical and manual CPR (38\% vs. $18 \%)$ with a two-sided test size of $5 \%$ and a power of $90 \%$, the trial required 114 subjects in each arm. Because of the availability of only 14 sets of LUCAS devices and a total of 46 emergency ambulances on the road in Singapore, a minimum of 375 patients needed to be recruited. However, owing to the unpredictability of cases of cardiac arrest being attended to by individual ambulances and the estimated annual incidence of about 1,400 OHCA patients attended to by the Emergency Ambulance Service, it was decided that the project should continue for a whole calendar year.

To compare pre-intervention characteristics between manual and mechanical CPR, we used Mann-Whitney $U$ test for continuous variables and chi-square or Fisher's exact tests for categorical variables. For intention-to-treat (ITT) and as-treated analyses, the survival $\mathrm{OR}$ and $\mathrm{Cl}$ of mechanical CPR for primary and secondary outcomes were calculated and tested in each presenting rhythm group. By further dividing the mechanical CPR group into early (LUCAS CPR started on-site) and late (LUCAS CPR started in ambulance) groups, the survival rates of each outcome were compared using chi-square tests.

\section{RESULTS}

A total of 1,274 patients were initially entered into the study. Of these, 1,191 fulfilled the eligibility criteria - 889 in the manual arm and 302 in the LUCAS arm. Of the 83 subjects who were excluded, 32 were under 21 years of age, 50 had trauma arrest and one patient's record was missing. The CONSORT diagram for the study is shown in Fig. 1.

\section{Patient characteristics}

The two treatment groups studied were similar for most characteristics, except that there were slightly more male patients and more witnessed cardiac arrests in the mechanical CPR group, and slightly more pulseless electrical activity patients in the manual CPR group (Table I). All the pulseless ventricular tachycardia (VT) patients $(n=13)$ were in the manual CPR group. There were no pulseless VT patients in the LUCAS arm. For all the patients in the LUCAS arm, manual CPR was initially performed with the mechanical device subsequently inserted. However, in 47 patients allocated to the LUCAS arm, the device was not applied for the following reasons: the patient was too small $(n=16,5.3 \%)$; the patient was too large $(n=7,2.3 \%)$; or the device was under servicing $(n=24,7.9 \%)$.

\section{Patient outcomes (by ITT analysis)}

From an ITT perspective, there were similar ROSC rates between the LUCAS and manual CPR arms (31.1\% vs. 29.2\%; OR 1.09, 95\% Cl 0.82-1.45; $p=0.537$; Table II). Similarly, all secondary outcome indicators were not significantly higher in the mechanical CPR arm. Among patients with ventricular fibrillation $(V F)$, there was no significant difference in patient outcomes with the use of mechanical or manual CPR (Table II). However, patients with pulseless VT (all in the manual CPR group) had the highest recorded primary and secondary outcome survival rates for all outcome indicators. For patients with an initial rhythm of asystole or pulseless electrical activity, there was no consistent 


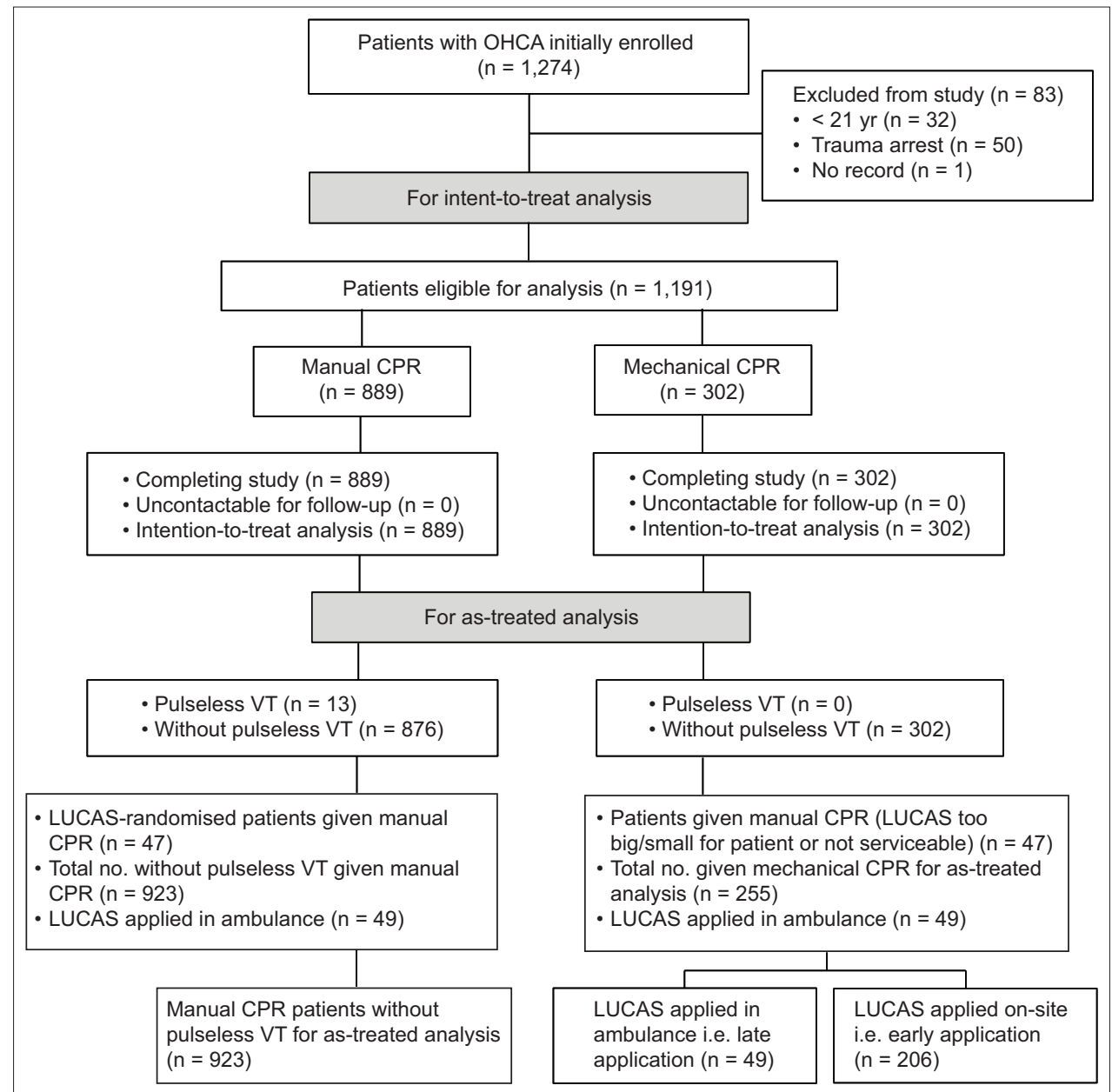

Fig. 1 CONSORT diagram for the MECCA study. CPR: cardiopulmonary resuscitation; OHCA: out-of-hospital cardiac arrest; VT: ventricular tachycardia

Table I. Pre-intervention characteristics of patients in the MECCA study.

\begin{tabular}{|c|c|c|c|c|c|c|}
\hline \multirow[t]{2}{*}{ Patient characteristic } & \multicolumn{3}{|c|}{ Intention-to-treat analysis } & \multicolumn{3}{|c|}{ As-treated analysis ${ }^{+}$} \\
\hline & $\begin{array}{c}\text { Manual CPR } \\
(n=889)\end{array}$ & $\begin{array}{l}\text { Mechanical } \\
\text { CPR }(n=302)\end{array}$ & p-value & $\begin{array}{c}\text { Manual CPR } \\
(\mathrm{n}=923)\end{array}$ & $\begin{array}{l}\text { Mechanical } \\
\text { CPR }(n=255)\end{array}$ & p-value \\
\hline Male gender & $571(64.2)$ & $221(73.2)$ & 0.005 & $591(64.0)$ & $191(74.9)$ & 0.001 \\
\hline Age* $^{*}(y r)$ & $66.9 \pm 15.7$ & $65.9 \pm 15.2$ & 0.323 & $67.1 \pm 15.9$ & $65.6 \pm 14.4$ & 0.128 \\
\hline No past medical history & $76(8.5)$ & $25(8.3)$ & 0.979 & $80(8.7)$ & $21(8.2)$ & 0.927 \\
\hline Witnessed cardiac arrest & $470(52.9)$ & $185(61.3)$ & 0.012 & $484(52.4)$ & $159(62.4)$ & 0.005 \\
\hline Bystander CPR & $284(31.9)$ & $96(31.8)$ & 1.000 & $290(31.4)$ & $81(31.8)$ & 0.977 \\
\hline \multicolumn{7}{|l|}{ Presenting rhythm } \\
\hline Ventricular fibrillation & $151(17.0)$ & $68(22.5)$ & 0.04 & $161(17.4)$ & $58(22.7)$ & 0.066 \\
\hline Ventricular tachycardia & $13(1.5)$ & $0(0)$ & - & - & - & - \\
\hline Asystole & $467(52.5)$ & $178(58.9)$ & 0.062 & $497(53.8)$ & $148(58.0)$ & 0.263 \\
\hline Pulseless electrical activity & $258(29.0)$ & $56(18.5)$ & $<0.001$ & $265(28.7)$ & $49(19.2)$ & 0.003 \\
\hline $\begin{array}{l}\text { Duration from collapse to start of } \\
\operatorname{CPR}^{*}(\min )\end{array}$ & $18.8 \pm 18.1$ & $17.5 \pm 13.9$ & 0.420 & $18.7 \pm 17.8$ & $17.8 \pm 14.6$ & 0.306 \\
\hline Hypertension & $314(35.3)$ & $114(37.7)$ & 0.49 & $323(35.0)$ & $102(40.0)$ & 0.162 \\
\hline Hyperlipidaemia & $35(3.9)$ & $13(4.3)$ & 0.911 & $36(3.9)$ & $12(4.7)$ & 0.691 \\
\hline Diabetes mellitus & $223(25.1)$ & $65(21.5)$ & 0.242 & $226(24.5)$ & $61(23.9)$ & 0.918 \\
\hline $\begin{array}{l}\text { Previous known ischaemic heart } \\
\text { disease }\end{array}$ & $262(29.5)$ & $89(29.5)$ & 1.000 & $263(28.5)$ & $83(32.5)$ & 0.238 \\
\hline
\end{tabular}

Data presented as no. (\%), unless otherwise stated. *Data presented as mean \pm standard deviation. + Excluding those with ventricular tachycardia. CPR: cardiopulmonary resuscitation 
Table II. Primary and secondary outcomes after manual and mechanical CPR (intention-to-treat analysis).

\begin{tabular}{|c|c|c|c|c|c|c|c|c|c|c|c|c|c|c|c|}
\hline \multirow[t]{2}{*}{ Outcome } & \multicolumn{3}{|c|}{ Ventricular fibrillation } & \multicolumn{3}{|c|}{ Ventricular tachycardia } & \multicolumn{3}{|c|}{ Asystole } & \multicolumn{3}{|c|}{ Pulseless electrical activity } & \multicolumn{3}{|c|}{ All patients } \\
\hline & $\begin{array}{c}\text { Manual } \\
\text { CPR } \\
(n=151)\end{array}$ & $\begin{array}{c}\text { LUCAS } \\
\text { CPR } \\
(n=68)\end{array}$ & $\begin{array}{c}\text { p-value } \\
\text { OR } \\
(95 \% \mathrm{Cl})\end{array}$ & $\begin{array}{c}\text { Manual } \\
\text { CPR } \\
(n=13)\end{array}$ & $\begin{array}{l}\text { LUCAS } \\
\text { CPR } \\
(n=0)\end{array}$ & $\begin{array}{c}\text { p-value } \\
\text { OR } \\
(95 \% \mathrm{Cl})\end{array}$ & $\begin{array}{c}\text { Manual } \\
\text { CPR } \\
(n=467)\end{array}$ & $\begin{array}{c}\text { LUCAS } \\
\text { CPR } \\
(n=178)\end{array}$ & $\begin{array}{c}\text { p-value } \\
\text { OR } \\
(95 \% \mathrm{Cl})\end{array}$ & $\begin{array}{c}\text { Manual } \\
\text { CPR } \\
(n=258)\end{array}$ & $\begin{array}{c}\text { LUCAS } \\
\text { CPR } \\
(n=56)\end{array}$ & $\begin{array}{c}\text { p-value } \\
\text { OR } \\
(95 \% \mathrm{Cl})\end{array}$ & $\begin{array}{c}\text { Manual } \\
\text { CPR } \\
(n=889)\end{array}$ & $\begin{array}{c}\text { LUCAS } \\
\text { CPR } \\
(n=302)\end{array}$ & $\begin{array}{c}\text { p-value } \\
\text { OR } \\
(95 \% \mathrm{Cl})\end{array}$ \\
\hline Rosc & $59(39.1)$ & $24(35.3)$ & $\begin{array}{c}0.595 \\
0.85 \\
(0.47,1.54)\end{array}$ & $8(61.5)$ & 0 & - & $103(22.1)$ & $44(24.7)$ & $\begin{array}{c}0.471 \\
1.16 \\
(0.77,1.74)\end{array}$ & $90(34.9)$ & $26(46.4)$ & $\begin{array}{c}0.105 \\
1.62 \\
(0.9,2.9)\end{array}$ & $260(29.2)$ & $94(31.1)$ & $\begin{array}{c}0.537 \\
1.09 \\
(0.82,1.45)\end{array}$ \\
\hline $\begin{array}{l}\text { 24-hr } \\
\text { survival }\end{array}$ & $33(21.9)$ & $13(19.1)$ & $\begin{array}{c}0.646 \\
0.85 \\
(0.41,1.73)\end{array}$ & $6(46.2)$ & 0 & - & $27(5.8)$ & $19(10.7)$ & $\begin{array}{c}0.031 \\
1.95 \\
(1.05,3.6)\end{array}$ & $34(13.2)$ & $8(14.3)$ & $\begin{array}{c}0.826 \\
1.10 \\
(0.48,2.52)\end{array}$ & $100(11.2)$ & $40(13.2)$ & $\begin{array}{c}0.352 \\
1.20 \\
(0.81,1.78)\end{array}$ \\
\hline $\begin{array}{l}\text { Survival to } \\
\text { discharge }\end{array}$ & $21(13.9)$ & $8(11.8)$ & $\begin{array}{c}0.666 \\
0.83 \\
(0.35,1.97)\end{array}$ & $5(38.5)$ & 0 & - & $1(0.2)$ & $2(1.1)$ & $\begin{array}{c}0.129 \\
5.30 \\
(0.48,58.77)\end{array}$ & 5 (1.9) & $3(5.4)$ & $\begin{array}{c}0.142 \\
2.86 \\
(0.66,12.35)\end{array}$ & $32(3.6)$ & $13(4.3)$ & $\begin{array}{c}0.579 \\
1.20 \\
(0.62,2.33)\end{array}$ \\
\hline $\begin{array}{l}\text { 30-day } \\
\text { survival }\end{array}$ & $20(13.2)$ & $8(11.8)$ & $\begin{array}{c}0.762 \\
0.87 \\
(0.36,2.09)\end{array}$ & $5(38.5)$ & 0 & - & $0(0)$ & $2(1.1)$ & $\begin{array}{c}0.022 \\
- \\
-\end{array}$ & $2(0.8)$ & $2(3.6)$ & $\begin{array}{c}0.091 \\
4.74 \\
(0.65,34.4)\end{array}$ & $27(3.0)$ & $12(4.0)$ & $\begin{array}{c}0.430 \\
1.32 \\
(0.66,2.64)\end{array}$ \\
\hline
\end{tabular}

Data presented as no. (\%), unless otherwise stated. Cl: confidence interval; CPR: cardiopulmonary resuscitation; OR: odds ratio; ROSC: return of spontaneous circulation

Table III. Primary and secondary outcomes after manual and mechanical CPR in patients without pulseless ventricular tachycardia (as-treated analysis).

\begin{tabular}{|c|c|c|c|c|c|c|c|c|c|c|c|c|}
\hline \multirow[t]{2}{*}{ Outcomes } & \multicolumn{3}{|c|}{ Ventricular fibrillation } & \multicolumn{3}{|c|}{ Asystole } & \multicolumn{3}{|c|}{ Pulseless electrical activity } & \multicolumn{3}{|c|}{ All patients } \\
\hline & $\begin{array}{c}\text { Manual } \\
\text { CPR } \\
(n=161)\end{array}$ & $\begin{array}{c}\text { LUCAS } \\
\text { CPR } \\
(n=58)\end{array}$ & $\begin{array}{c}\text { p-value } \\
\text { OR } \\
(95 \% \mathrm{Cl})\end{array}$ & $\begin{array}{c}\text { Manual } \\
\text { CPR } \\
(n=497)\end{array}$ & $\begin{array}{c}\text { LUCAS } \\
\text { CPR } \\
(n=148)\end{array}$ & $\begin{array}{c}\text { p-value } \\
\text { OR } \\
(95 \% \mathrm{Cl})\end{array}$ & $\begin{array}{c}\text { Manual } \\
\text { CPR } \\
(n=265)\end{array}$ & $\begin{array}{c}\text { LUCAS } \\
\text { CPR } \\
(n=49)\end{array}$ & $\begin{array}{c}\text { p-value } \\
\text { OR } \\
(95 \% \mathrm{Cl})\end{array}$ & $\begin{array}{c}\text { Manual } \\
\text { CPR } \\
(n=923)\end{array}$ & $\begin{array}{c}\text { LUCAS } \\
\text { CPR } \\
(n=255)\end{array}$ & $\begin{array}{c}\text { p-value } \\
\text { OR } \\
(95 \% \mathrm{Cl})\end{array}$ \\
\hline ROSC & $61(37.9)$ & $22(37.9)$ & $\begin{array}{c}0.995 \\
1.00 \\
(0.54,1.86)\end{array}$ & $107(21.5)$ & $40(27.0)$ & $\begin{array}{c}0.162 \\
1.35 \\
(0.89,2.06)\end{array}$ & $90(34.0)$ & $26(53.1)$ & $\begin{array}{c}0.011 \\
2.20 \\
(1.19,4.07)\end{array}$ & $258(28.0)$ & $88(34.5)$ & $\begin{array}{c}0.042 \\
1.36 \\
(1.01,1.83)\end{array}$ \\
\hline $\begin{array}{l}\text { 24-hr } \\
\text { survival }\end{array}$ & $34(21.1)$ & $12(20.7)$ & $\begin{array}{c}0.945 \\
0.97 \\
(0.47,2.04)\end{array}$ & $30(6.0)$ & $16(10.8)$ & $\begin{array}{c}0.048 \\
1.89 \\
(1,3.57)\end{array}$ & $34(12.8)$ & $8(16.3)$ & $\begin{array}{c}0.51 \\
1.33 \\
(0.57,3.07)\end{array}$ & $98(10.6)$ & $36(14.1)$ & $\begin{array}{c}0.119 \\
1.38 \\
(0.92,2.09)\end{array}$ \\
\hline $\begin{array}{l}\text { Survival to } \\
\text { discharge }\end{array}$ & $21(13.0)$ & $8(13.8)$ & $\begin{array}{c}0.885 \\
1.07 \\
(0.44,2.56)\end{array}$ & $1(0.2)$ & $2(1.4)$ & $\begin{array}{c}0.071 \\
6.79 \\
(0.61,75.46)\end{array}$ & $5(1.9)$ & $3(6.1)$ & $\begin{array}{c}0.084 \\
3.39 \\
(0.78,14.68)\end{array}$ & $27(2.9)$ & $13(5.1)$ & $\begin{array}{c}0.090 \\
1.78 \\
(0.91,3.51)\end{array}$ \\
\hline $\begin{array}{l}\text { 30-day } \\
\text { survival }\end{array}$ & $20(12.4)$ & $8(13.8)$ & $\begin{array}{c}0.789 \\
1.13 \\
(0.47,2.72)\end{array}$ & $0(0)$ & $2(1.4)$ & $\begin{array}{c}0.009 \\
\operatorname{lnf} \\
(\mathrm{NaN}, \operatorname{Inf})\end{array}$ & $2(0.8)$ & $2(4.1)$ & $\begin{array}{c}0.057 \\
5.60 \\
(0.77,40.71)\end{array}$ & $22(2.4)$ & $12(4.7)$ & $\begin{array}{c}0.050 \\
2.02 \\
(0.99,4.14)\end{array}$ \\
\hline
\end{tabular}

Data presented as no. (\%), unless otherwise stated. Cl: confidence interval; CPR: cardiopulmonary resuscitation; Inf: infinity; NaN: not a number; OR: odds ratio; ROSC: return of spontaneous circulation 


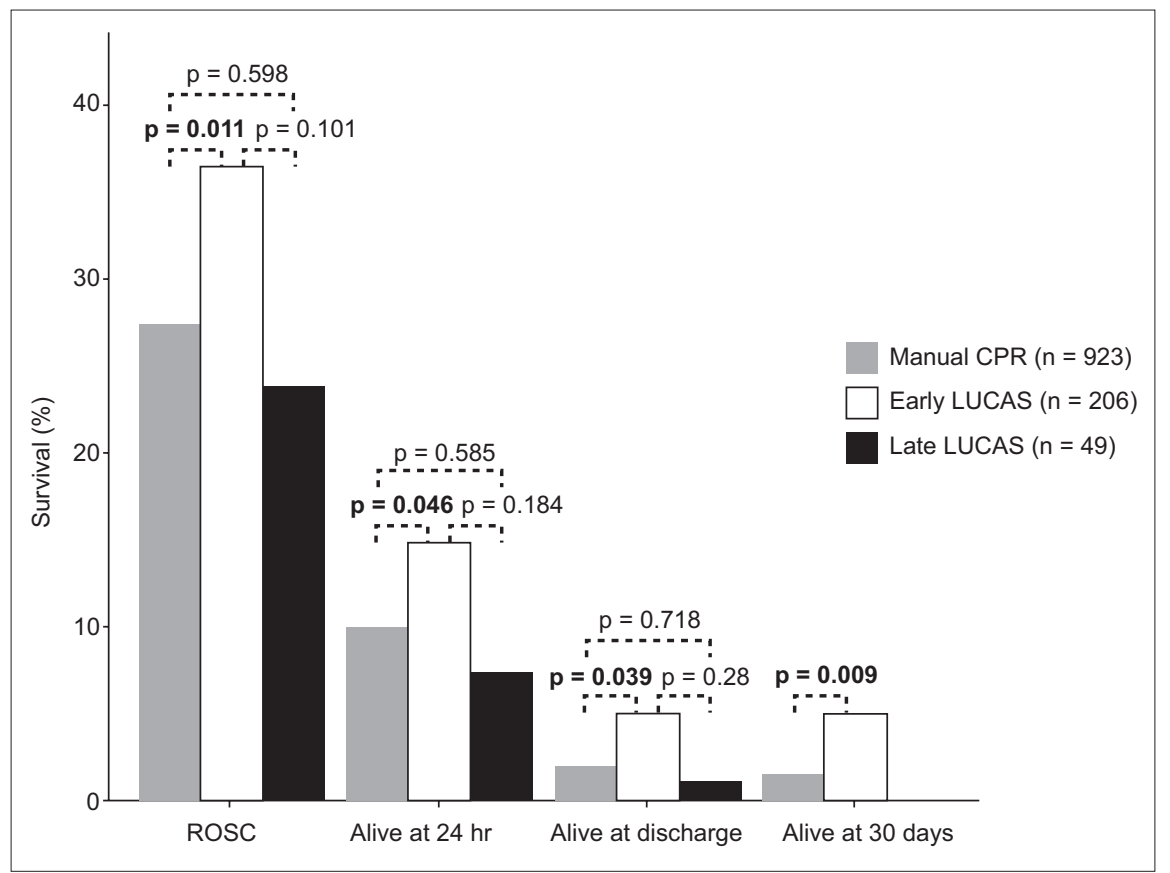

Fig. 2 Graph shows as-treated outcomes of manual cardiopulmonary resuscitation (CPR) (excluding ventricular tachycardia patients), and early- and late-LUCAS applications. The p-values in bold are significant. ROSC: return of spontaneous circulation

statistical advantage with mechanical CPR, even though survival numbers were greater with the LUCAS 2.

\section{Patient outcomes (by as-treated analysis)}

The following groups of patients were identified as having not complied with treatment assignment: (a) patients in whom the mechanical device could not be fitted $(n=47)$ due to size issues or because the device was not serviceable, and were thus provided with only manual CPR; (b) patients who received delayed LUCAS CPR, i.e. initiated in the ambulance rather than on-site $(n=49$ in the mechanical CPR group).

In addition, patients with pulseless VT were wholly in the manual group, resulting in a potential survival advantage for this group over those in the mechanical arm; thus, these patients ( $n=13$ ) were excluded from the as-treated analysis. As a result, 923 patients were provided only manual CPR and 255 were treated with LUCAS CPR (Table I, Fig. 1). The improvements in ROSC and 30-day survival for those treated with mechanical CPR were evident (Table III). Using multiple logistic regression modelling (considering witnessed arrests, bystander CPR, presenting rhythm and duration of cardiac arrest), the adjusted OR for survival with LUCAS CPR was $1.32(p=0.081)$ compared with manual CPR.

Of the 255 patients who received LUCAS CPR, 206 (80.8\%) had the device applied early and promptly at the site of collapse, while $49(19.2 \%)$ patients had it applied only upon reaching the ambulance. In the former group, 107 (42.0\%) patients had the LUCAS 2 device applied within one minute of initiating manual CPR and 99 (38.8\%) within the next four minutes. For those whose device was applied in the ambulance, 15 (5.9\%) received it within ten minutes and 34 (13.3\%) more than ten minutes after manual CPR was started. The primary and secondary outcomes of these groups are presented in Fig. 2. Outcome rates in the manual, early-LUCAS and late-LUCAS groups were - ROSC: $28.0 \%, 36.9 \%$ and $24.5 \%$; 24 -hour survival: $10.6 \%, 15.5 \%$ and $8.2 \%$; survival to discharge: $2.9 \%, 5.8 \%$ and $2.0 \%$; and 30 -day survival: $2.4 \%, 5.8 \%$ and $0.0 \%$, respectively.

These results indicate that, compared to the use of manual CPR only, the survival effect of the LUCAS CPR device was significantly greater when applied early, on-site, before the patient is moved into the ambulance, and within five minutes of starting resuscitation. Using multiple logistic regression modelling and adjusting for witnessed arrests, bystander CPR, presenting rhythm and duration of cardiac arrest, the adjusted OR for early LUCAS vs. manual CPR was $1.47(p=0.026)$.

\section{DISCUSSION}

The MECCA study showed no statistically significant advantage for the LUCAS 2 mechanical CPR device over standard manual CPR. Our findings are similar to those of large studies conducted over the last few years that examined the outcomes of OHCA from an ITT perspective when mechanical CPR devices were compared to manual CPR. The ITT analysis, assuming optimal operational implementation of the device, may ignore existing operational limitations that may influence statistical outcomes and may not demonstrate the true value of the device if used early. For time-critical conditions such as cardiac arrest, if caregivers strictly adhere to device protocols (such as applying the device on-site and as early as possible), the potential benefits, if any, may become apparent.

This is especially relevant for the provision of the best level of $\mathrm{CPR}$ in the community. For OHCA, there is a need for a consistent level of good-quality, early CPR with minimal interruptions until achievement of ROSC. Manual CPR in out-of-hospital 
and in-hospital environments is beset with many quality issues and frequent interruptions. Rescuer fatigue within the first few minutes affects the quality of manual compressions, resulting in suboptimal coronary perfusion pressures with greater likelihood of poorer outcomes. Mechanical CPR devices were introduced to specifically address the various interruptions in provision of good-quality manual CPR. Operational challenges, such as ambulance crew not carrying it to the site because of the heavy weight of the device, mechanical faults, noncompliance to protocols by the crew and delays in the use of the device, may affect outcomes. It would appear intuitive that mechanical CPR devices, if efficiently implemented for OHCA, can potentially result in better clinical outcomes, especially if applied early. This can significantly improve outcomes ${ }^{(21)}$ and would be in line with the chain of survival for cardiac arrest, which emphasises 'early' institution of the various links.

In addition to the ITT analysis, we conducted an as-treated analysis, which gave an estimate of likely outcomes if our OHCA care processes were more efficient. We also documented adherence data for mechanical CPR, such as time to application and place of application of device. The LINC trial reported a $24 \%$ rate of non-adherence to protocol. ${ }^{(20)}$ It is through such documentation that one begins to better appreciate the potential true treatment effects of mechanical CPR devices. Since the astreated analysis suggests better survival outcomes with early use of the LUCAS 2 device than with standard manual CPR, future studies in this area of care would need to focus on the efficient use of the device, rather than on its uncontrolled, late or unguided application, to better determine the potential value of mechanical CPR in the management of OHCA.

In this study, we applied the LUCAS 2 device in the continuous CPR mode with interposed ventilations at every tenth piston compression rather than in the 30:2 mode. This helped achieve continuous chest compressions and ventilations that were closer to the regular physiological mode than the very artificial 30:2 mode originally meant for lay one-person CPR. The mechanical device takes the place of one person, freeing the ambulance staff of a pair of hands to perform other tasks such as interposed ventilations, defibrillation and administration of drugs to enhance the performance of circulation, without interrupting circulation and coronary perfusion.

This study was not originally powered to conduct analyses of the early use of the LUCAS 2 device vs. late use or manual CPR. The trial had originally presumed complete compliance in instituting early use of the mechanical CPR device on-site in all instances. The resulting noncompliance allowed comparison of true early use of mechanical CPR with manual CPR and late use.

Many factors may influence the decision by the ambulance crew to initiate the use of a mechanical device in their vehicle rather than in full view of the public. These include the additional weight of the mechanical device to be carried by the crew, having to put up with members of the public questioning the use of what may seem a frightening piston device pumping away at the chest, and unclear messages by the emergency caller, resulting in the call centre not recognising a cardiac arrest call in the first instance. While these issues may need to be addressed, due consideration should be given to enable the ambulance team to carry the mechanical CPR device to the patient in every instance, so as to minimise delay in application. Future efforts to reduce the size and weight of mechanical CPR devices may allow for greater portability and earlier application. Furthermore, current designs of mechanical CPR devices may not fit every cardiac arrest patient. The $7.6 \%$ of patients in the current study who could not fit into the LUCAS 2 device was higher than the $5.0 \%$ reported in the LINC trial, ${ }^{(20)}$ possibly owing to the generally smaller size of Asian patients compared to those who participated in the LINC trial.

In addition, one should consider whether future studies need to separate the analysis of patients with an initial rhythm of pulseless VT and those with VF. This is because the former tends to have a far higher survival rate, and thus analysis of the two types of rhythms together may affect patient outcome results and understanding of VF patients.

The current study had a few limitations. Firstly, cerebral performance category scores were not measured for survivors, and thus not reported. Secondly, we have not yet determined the CPR fraction or adequacy of compressions achieved for both the manual and mechanical arms as a marker of the quality of CPR provided. Thirdly, it was impossible to blind the ambulance crew and ED team to the treatment provided. Fourthly, we could not standardise the resuscitation and treatment protocols after the patients were admitted to the ED of the various hospitals. Finally, the limited number of available mechanical CPR devices did not allow larger numbers to be randomised to the mechanical CPR arm. Despite the limitations, the results of our study provide the direction for future research efforts.

In conclusion, mechanical CPR devices have a role to play for patients with $\mathrm{OHCA}$, at least in ensuring consistent performance of good-quality chest compressions. Although the MECCA study was unable to show a significant survival benefit with the LUCAS 2 device compared to manual CPR, the as-treated analysis suggested better survival outcomes if the device was applied early, rather than late or with standard manual CPR.

\section{ACKNOWLEDGEMENTS}

The authors acknowledge the tremendous support to the study provided by the management of the Singapore Civil Defence Force and the crew of the Emergency Ambulance Service.

\section{REFERENCES}

1. Travers AH, Perkins GD, Berg RA, et al; Basic Life Support Chapter Collaborators. Part 3: adult basic life support and automated external defibrillation: 2015 International Consensus on Cardiopulmonary Resuscitation and Emergency Cardiovascular Care Science with Treatment Recommendations. Circulation 2015; 132(16 suppl 1):S51-83.

2. Eng Hock Ong M, Chan YH, Anantharaman V, et al. Cardiac arrest and resuscitation epidemiology in Singapore (CARE I study). Prehosp Emerg Care 2003; 7:427-33.

3. Sugerman NT, Edelson DP, Leary M, et al. Rescuer fatigue during actual inhospital cardiopulmonary resuscitation with audiovisual feedback: a prospective multicenter study. Resuscitation 2009; 80:981-4.

4. Halperin HR, Paradis N, Ornato JP, et al. Cardiopulmonary resuscitation with a novel chest compression device in a porcine model of cardiac arrest: improved hemodynamics and mechanisms. J Am Coll Cardiol 2004; 44:2214-20.

5. Timerman S, Cardoso LF, Ramires JA, Halperin H. Improved hemodynamic 
performance with a novel chest compression device during treatment of inhospital cardiac arrest. Resuscitation 2004; 61:273-80.

6. Ikeno F, Kaneda H, Hongo Y, et al. Augmentation of tissue perfusion by a novel compression device increases neurologically intact survival in a porcine model of prolonged cardiac arrest. Resuscitation 2006; 68:109-18.

7. Duchateau FX, Gueye P, Curac S, et al. Effect of the AutoPulse automated band chest compression device on hemodynamics in out-of-hospital cardiac arrest resuscitation. Intensive Care Med 2010; 36:1256-60.

8. Ong ME, Ornato JP, Edwards DP, et al. Use of an automated, load-distributing band chest compression device for out-of-hospital cardiac arrest resuscitation. JAMA 2006; 295:2629-37.

9. Hock Ong ME, Fook-Chong S, Annathurai A, et al. Improved neurologically intact survival with the use of an automated, load-distributing band chest compression device for cardiac arrest presenting to the emergency department. Crit Care 2012; 16:R144.

10. Steen S, Liao Q, Pierre L, Paskevicius A, Sjöberg T. Evaluation of LUCAS, a new device for automatic mechanical compression and active decompression resuscitation. Resuscitation 2002; 55:285-99.

11. Rubertsson S, Karlsten R. Increased cortical cerebral blood flow with LUCAS; a new device for mechanical chest compressions compared to standard external compressions during experimental cardiopulmonary resuscitation. Resuscitation. 2005; 65:357-63.

12. Axelsson C, Karlsson T, Axelsson AB, Herlitz J. Mechanical active compressiondecompression cardiopulmonary resuscitation (ACD-CPR) versus manual CPR according to pressure of end tidal carbon dioxide $(\mathrm{P}(\mathrm{ET}) \mathrm{CO} 2)$ during $\mathrm{CPR}$ in out-of-hospital cardiac arrest (OHCA). Resuscitation 2009; 80:1099-103.

13. Wagner $\mathrm{H}$, Terkelsen $\mathrm{C}$ ), Friberg $\mathrm{H}$, et al. Cardiac arrest in the catheterisation laboratory: a 5-year experience of using mechanical chest compressions to facilitate $\mathrm{PCl}$ during prolonged resuscitation efforts. Resuscitation 2010; 81:383-7.

14. Wik L, Kiil S. Use of an automatic mechanical chest compression device (LUCAS) as a bridge to establishing cardiopulmonary bypass for a patient with hypothermic cardiac arrest. Resuscitation 2005; 66:391-4.

15. Holmström P, Boyd J, Sorsa M, Kuisma M. A case of hypothermic cardiac arrest treated with an external chest compression device (LUCAS) during transport to re-warming. Resuscitation 2005; 67:139-41.

16. Larsen AI, Hjørnevik AS, Ellingsen CL, Nilsen DW. Cardiac arrest with continuous mechanical chest compression during percutaneous coronary intervention. A report on the use of the LUCAS device. Resuscitation 2007; 75:454-9.

17. Larsen Al, Hjørnevik $\AA$, Bonarjee V, et al. Coronary blood flow and perfusion pressure during coronary angiography in patients with ongoing mechanical chest compression: a report on 6 cases. Resuscitation 2010; 81:493-7.

18. Axelsson C, Nestin J, Svensson L, Axelsson AB, Herlitz J. Clinical consequences of the introduction of mechanical chest compression in the EMS system for treatment of out-of-hospital cardiac arrest-a pilot study. Resuscitation 2006; 71:47-55.

19. Wik L, Olsen JA, Persse D, et al. Manual vs. integrated automatic loaddistributing band CPR with equal survival after out of hospital cardiac arrest. The randomized CIRC trial. Resuscitation 2014; 85:741-8.

20. Rubertsson S, Lindgren E, Smekal D, et al. Mechanical chest compressions and simultaneous defibrillation vs conventional cardiopulmonary resuscitation in out-of-hospital cardiac arrest: the LINC randomized trial. JAMA 2014; 311:53-61.

21. Janata A, Bayegan K, Sterz F, et al. Limits of conventional therapies after prolonged normovolemic cardiac arrest in swine. Resuscitation 2008; 79:133-8. 